\title{
RF-Induced Unintended Stimulation for Implantable Medical Devices in MRI
}

\author{
James E. Brown, Rui Qiang, Paul J. Stadnik, Larry J. Stotts, \\ and Jeffrey A. Von Arx
}

\section{Introduction}

As the preferred imaging modality for soft tissue imaging, there has been significant interest in recent years to provide access to magnetic resonance imaging (MRI) to patients with active implantable medical devices (AIMDs). Historically, these patients have been denied access to this important diagnostic tool due to several potentially hazardous interactions with AIMDs [1]. Examples of AIMDs include pacemakers, cochlear implants, implantable glucose monitors, spinal cord stimulators, and deep brain stimulators.

AIMD manufacturers, in cooperation with MRI manufacturers, regulatory bodies, and academia, have developed a technical specification which outlines test methods for assessing the risk of several hazards [2]. In test methods, hazards are separated according to the field component (static, gradient, or radio frequency (RF)), and conservative test conditions are derived to stress the device in a laboratory setting beyond what is possible in the complex MR environment.

Among the potential hazards of MRI for patients with active implantable medical device is RF-induced rectified voltage. RF energy incident on the device may be rectified by internal active components and cause unintended stimulation of tissue near the device electrodes. In order to assess the risk to the patient, device manufacturers use computational human models to quantify the incident RF on the device and perform benchtop testing to determine the likelihood of unintended stimulation.

For a cardiac implantable electronic device (CIED) such as a pacemaker, if this rectified voltage exceeds the patient's physiological threshold, it could induce unintended cardiac stimulation (UCS) leading to tachycardia. A standard test for leaded CIEDs is presently being developed to quantify the risk of UCS for these

J. E. Brown $(\bowtie) \cdot$ R. Qiang · P. J. Stadnik · L. J. Stotts · J. A. Von Arx

Micro Systems Engineering, Inc., Lake Oswego, OR, USA

e-mail: james.brown@biotronik.com 
devices [3]. The safety assessment for RF-induced UCS utilizes computational human models (CHMs).

In this work, the general process of using CHMs for the assessment of RF-induced unintended stimulation is discussed in the next section. Then, the process is applied to a pacemaker as an example in the following section for the specific hazard of UCS. Next, the impact of the model development on the results of this analysis is discussed. Finally, the overall impact of this work and areas which should be considered for future work are presented.

\section{Evaluation of RF-Induced Unintended Stimulation Using Computational Human Models}

There are many advantages of using CHMs for risk-based safety assessments for AIMD in MRI [4, 5]. The RF-induced energy incident on a leaded AIMD is typically calculated through the use of the well-known transfer function method $[6,7]$ as

$$
V_{D U T}=A \int_{0}^{L} S(\tau) \cdot E_{\text {tan }}(\tau) d \tau .
$$

Here, $V_{D U T}$ is the voltage at the AIMD under test, $S$ is the transfer function, and $E_{\text {tan }}$ is portion of the incident electric field which is tangent to the lead pathway. These lead models are combined with RF field distributions within CHMs derived through electromagnetic simulation to conservatively estimate the induced RF level.

The variability of the predicted induced RF level for leaded devices is quite large due to the presence of resonant phenomena. These resonance effects have been extensively studied in the literature [8-11], usually in terms of RF-induced heating, but the same principle applies to the RF level induced at the implantable pulse generator (IPG). RF heating is evaluated at the distal end of the lead, while RF-induced energy is quantified at the proximal end, but otherwise the phenomena are very much related. Both may be influenced differently by the terminating impedance at the IPG [12].

\subsection{3-D Field Distribution Within the CHM}

A library of CHMs spanning the population in terms of height and BMI in different body positions, MR coils, landmark positions is used to study the distribution of expected electromagnetic fields along the lead pathway. The overall procedure is shown in the flowchart of Fig. 1. 


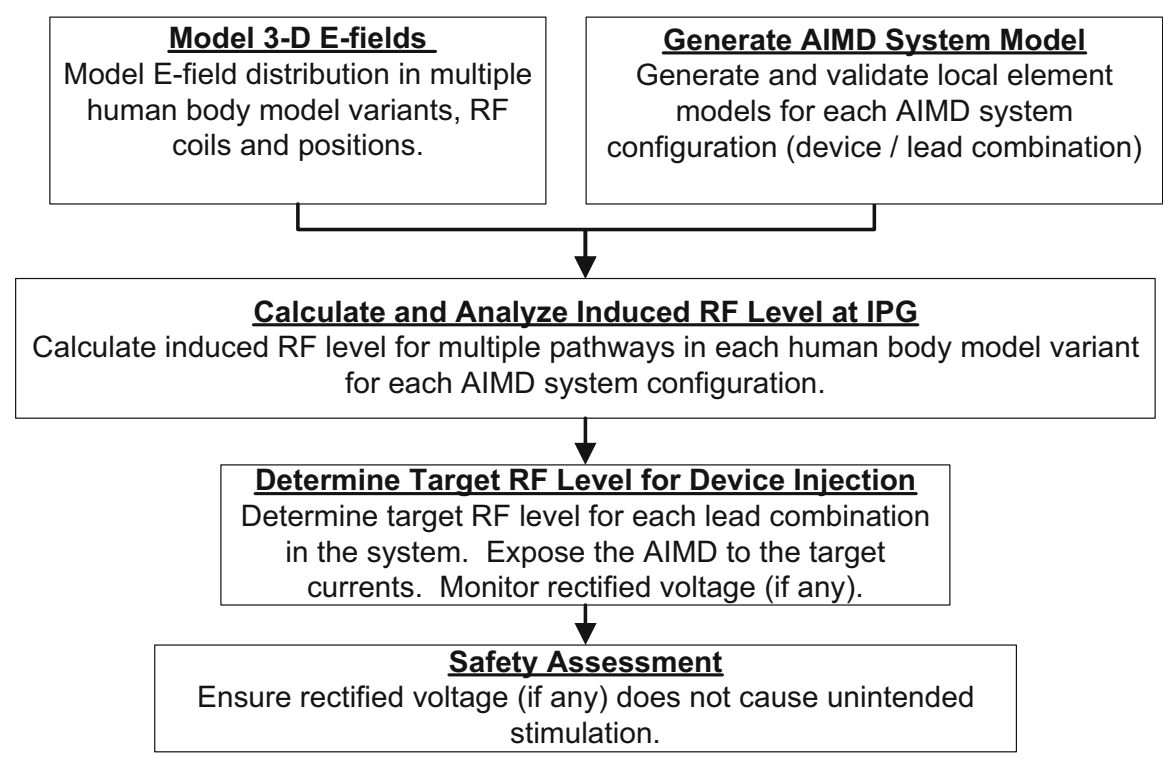

Fig. 1 Flow chart illustrating workflow for the assessment of protection from harm to the patient caused by RF-induced unintended stimulation

\subsection{AIMD System Model}

Lead models are developed numerically or experimentally [13-17], in one or more tissue-simulating media (TSM). The homogenous TSM should be chosen to accurately compute the induced RF level once the transfer function is applied in the human body (via CHM). The variability of computed RF level with the TSM used during lead model development is discussed in Sect. 4.

The geometric accuracy of the CHM along the lead trajectory, including the continuity of organs through which the leads are placed, is paramount to the accuracy of the model. Variations in critical parameters such as surrounding anatomy and tissue parameters must be included in the set of simulations used to generate the worst-case predicted RF-induced energy.

\subsection{Assessment of AIMD Response}

A risk assessment is then performed to determine the set of target exposure values for a benchtop RF injection test. Due to the nonlinear nature of rectified voltage, the test must be performed using the large-signal RF level (i.e., results cannot be scaled). Observed rectified voltage can be compared with a patient's expected physiological response, which is dependent on the specific tissues that are near the electrodes. 
As an example of the evaluation of this hazard for a specific implantable device, this procedure is detailed for a pacemaker system in the next section. Analogous methods may be applied to extend the analysis to other types of AIMDs, including spinal cord stimulators and deep brain stimulators.

\section{Approach Applied to a Pacemaker System}

In order to apply the procedure from Sect. 2 to CIEDs, the incident fields in the CHMs are extracted for clinically relevant pathways. An example orientation for a dual-lead pacemaker system is shown in Fig. 2.

Additionally, lead models must be developed for all lead combinations which are to be evaluated. The induced RF level for multiple lead systems has been observed to be higher than for single lead systems [3].

A probability distribution of induced RF levels is created using each lead model and set of extracted fields from the library of CHMs. Additionally, if the device includes an RF antenna, the induced voltage on the antenna must be quantified $[18,19]$. Then, benchtop testing is performed to expose the pacemaker to a suitable range of target RF levels so as to appropriately characterize the risk to the patient. Any observed rectified voltage can then be compared with the statistical likelihood that the waveform will cause cardiac stimulation, for example, through the strength duration curve [19]. In [3], the criterion is that the probability of unintended cardiac stimulation, $P(U C S)$, shall be less than 1 in 10,000 . This probability is assessed by computing the integral

Fig. 2 Example orientation of a dual-chamber pacemaker in the human body

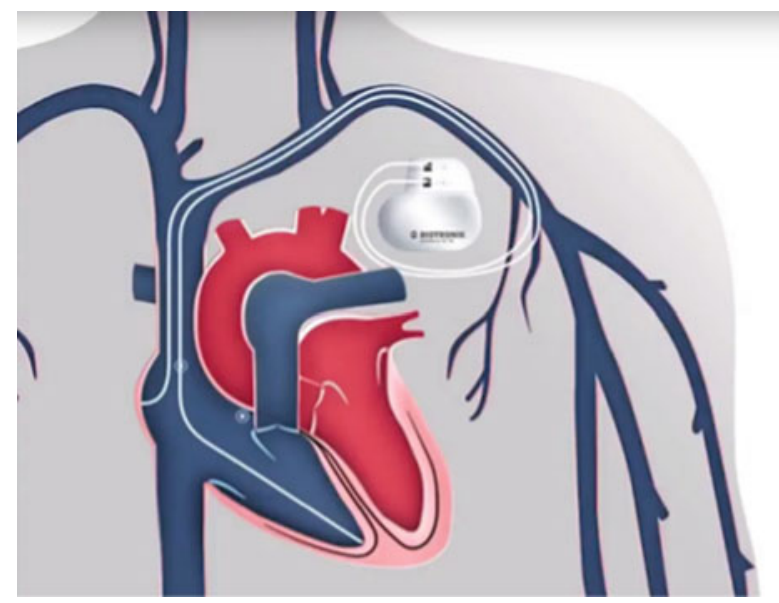


Fig. 3 Computing the probability of unintended stimulation

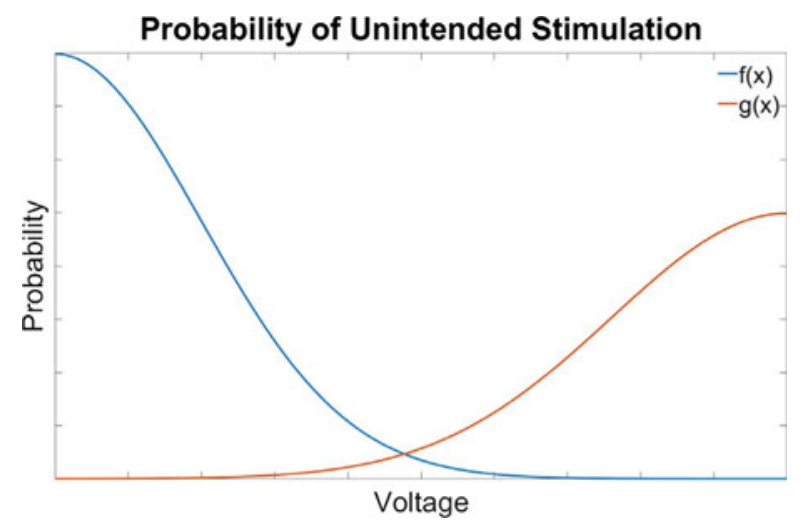

Fig. 4 The strengthduration curve

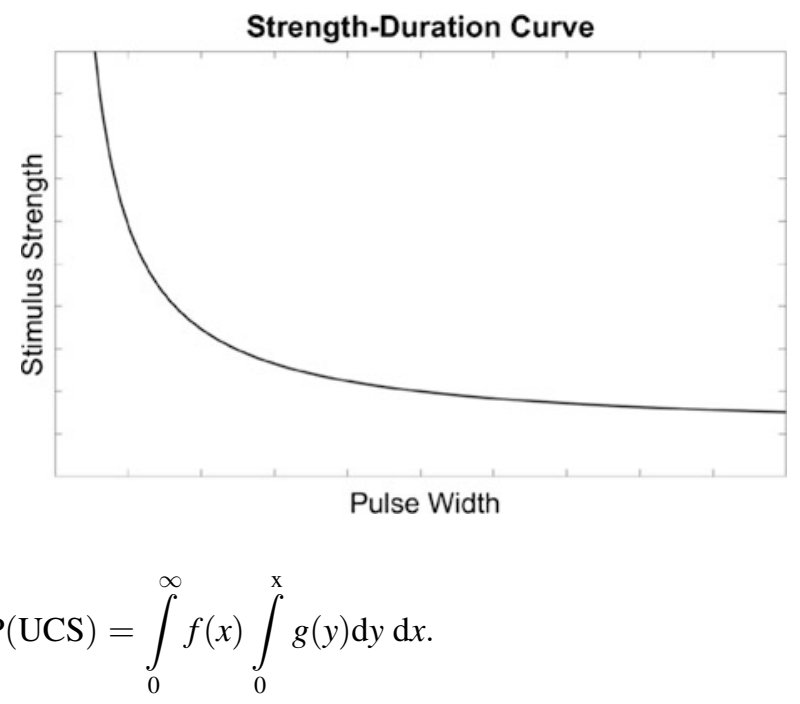

Here, $f(x)$ is the probability that a given rectified voltage is induced during the MRI, and $g(x)$ is the probability that a given voltage is the minimum required to stimulate the patient's cardiac tissue. This is illustrated in Fig. 3, where the y-axis is probability and the $\mathrm{x}$-axis is voltage. The voltage is the voltage monitored across a tissue-simulating resistor and thus is directly proportional to current. Current is a surrogate for charge, which is the true figure of merit for evaluating the probability of stimulation.

The pulse width of the rectified voltage is important to assess the probability of unintended stimulation. The well-known strength duration curve [20], shown in Fig. 4, can be used to determine the dependence of the analysis on rectified pulse width. 


\section{Variability of Induced RF Level}

The computed RF level is dependent both on the CHMs being used and the tissuesimulating medium (TSM) used during lead model development. This topic has been investigated extensively for RF-induced heating in the literature [21-25]. Here, we include some discussion of the impact of the TSM on the computation of the induced RF level, which would in the end impact the assessment of the probability of unintended stimulation.

A basic structure approximating an implanted medical device lead is described in [2] as SAIMD-1. Here, the lead length is changed to $45 \mathrm{~cm}$ in order to better represent a pacemaker lead. The device geometry is shown in Fig. 5.

The incorporation of the lead model uses a method similar to [7], where a voltage is introduced between the wire and device ground, and the transfer function is then proportional to the current distribution on the wire. The surrounding medium is swept through a range of dielectric constants and conductivities. For example, the transfer function magnitude for $0.2 \mathrm{~S} / \mathrm{m}, 1.2 \mathrm{~S} / \mathrm{m}$, and $2.2 \mathrm{~S} / \mathrm{m}$ are given in Fig. 6.

From these plots, it can be observed that a lower dielectric constant of TSM pushes the lead below resonance. The resonant behavior is more pronounced for the higher conductivity of TSM due to wavelength compression effects.

Naturally, these lead models will lead to different predictions for the induced RF level in the patient and thus varied risk of unintended stimulation. The lead model is inserted into the VHP-Female v3.0 [26] along a representative pathway, and the

Fig. 5 Cross section of the insulated wire. The wire is $0.8 \mathrm{~mm}$ diameter with insulation of $0.5 \mathrm{~mm}$ wall thickness
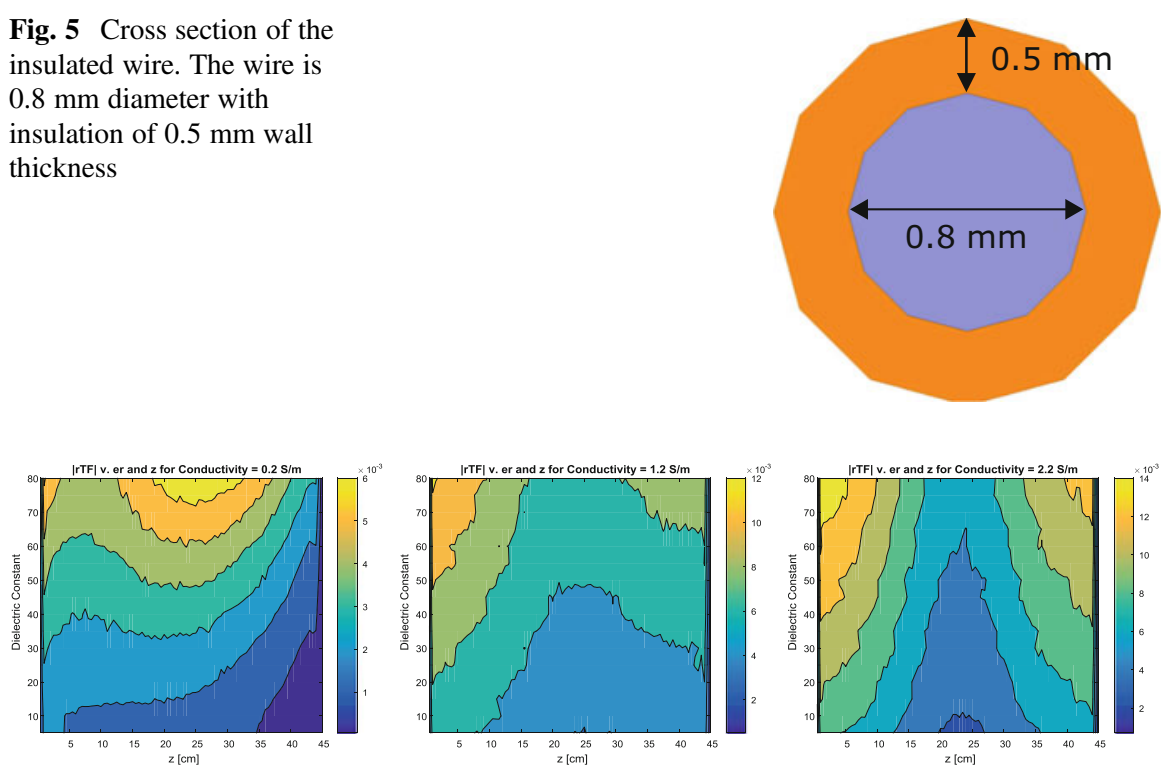

Fig. 6 Transfer function magnitude (v. length) versus dielectric constant of the TSM for three selected TSM conductivities 


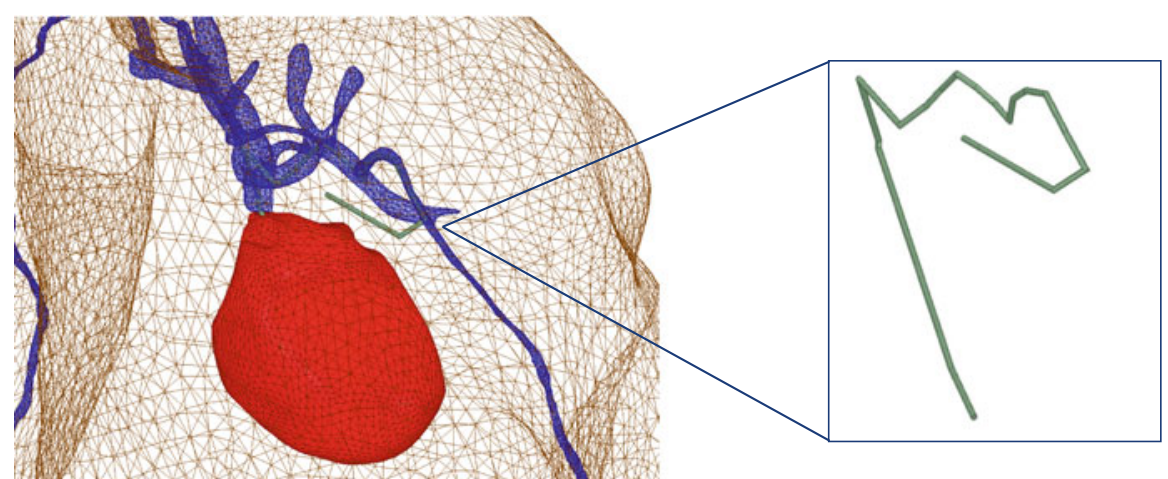

Fig. 7 Representative cardiac pathway within VHP-female v3.0 (only selected anatomical features are visible)

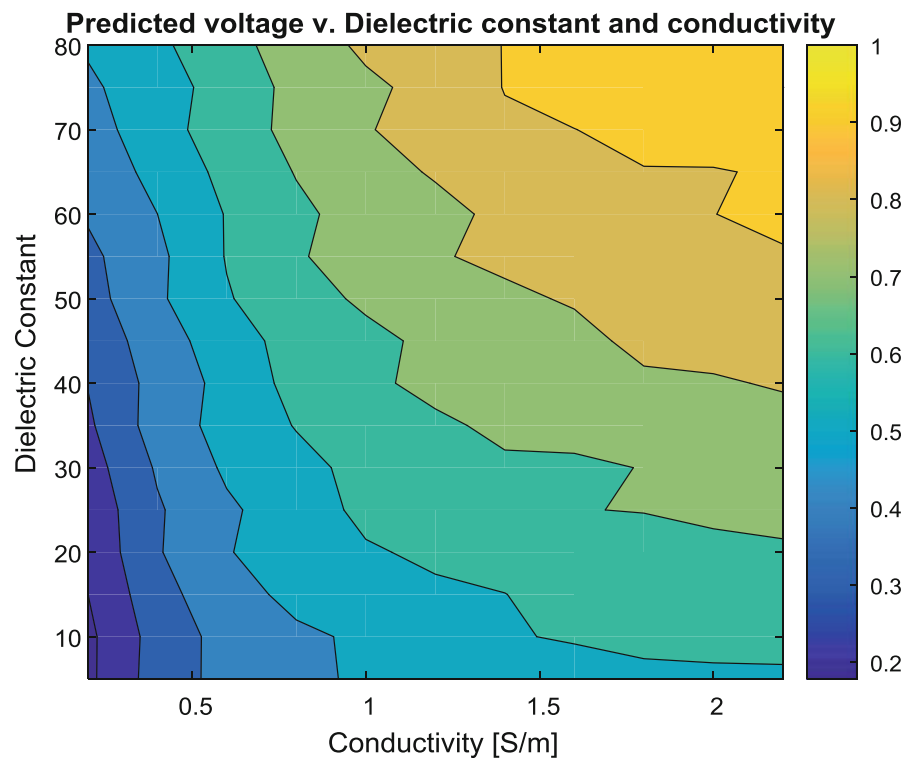

Fig. 8 Predicted in vivo MRI RF-induced voltage as a function of TSM dielectric constant and conductivity

incident field values are extracted. The pathway and its insertion into the model are shown in Fig. 7.

For this pathway, the RF-induced energy at the device is calculated for each of the transfer functions with different TSM. The normalized voltages are shown in Fig. 8. Notably, the higher the dielectric constant and the conductivity, the higher the predicted induced RF level which must be used for device testing. Care should be taken that an appropriate TSM is chosen such that the device test is conservative 
without wildly overexposing the hardware to RF levels beyond what is expected in the clinical environment.

\section{Discussion}

Manufacturers of AIMDs use CHMs in order to protect the patient from harm due to the risk of RF-induced unintended stimulation. The use of CHMs enables the investigation of millions of scenarios of scan parameters, patient sizes and anatomies, and MR system technologies. Therefore, CHMs allow AIMD manufacturers to quantify low probability events such as (for cardiac implantable electronic devices) UCS that represent a high risk to the patient.

The predicted RF-induced energy incident on the device is used for benchtop testing in order to convert a probability of an incident RF level to a probability of rectifed voltage. This probability distribution is combined with the probability of stimulating the patient through a physiological model.

It is notable that the predicted RF level is model dependent; thus, an appropriate TSM should be used. A TSM that shows overly resonant lead behavior will overpredict the incident RF level and thus overestimate the risk to the patient of unintended stimulation. This would result in denying access to MRI to patients that could benefit from this diagnostic tool.

This work explored the application of this procedure to the example of a pacemaker, where unintended stimulation of cardiac tissue could result in tachycardia. For other classes of devices, this could mean anything from muscular discomfort, to recruitment of unintended fibers in the spinal column, to significant impact related to stimulating tissue within the deep brain.

\subsection{Future Work}

The results presented here are for a single CHM and one device orientation within the MRI field. The procedures are valid for the extension to a set of CHMs and for many devices, enabling the safety assessment of AIMDs by identifying low-probability worst-case-based assessments.

Future work in this area could allow for co-simulation of the incident RF field and circuit model of internal device hardware, especially active components that could rectify the incident RF level. One option for this is to combine the circuit model with a Tier 4 model [2] of the realistic medical device lead geometry in the patient, with the MR coil. Additionally, computational methods have been shown to be effective in evaluating a range of shimming conditions [27, 28].

Further, the incorporation of a physiological model into the electromagnetic CHM could eliminate the need for clinical data to assess the relationship between rectified waveforms and the probability of stimulating tissue. An additional benefit 
of this technique would be the evaluation of waveforms not often used in the clinical setting, including cross-channel rectification which may not mimic a therapy waveform.

\section{References}

1. Kalin, R., \& Stanton, M. S. (Apr 2005). Current clinical issues for MRI scanning of pacemaker and defibrillator patients. Pacing and Clinical Electrophysiology, 28(4), 326-328.

2. ISO/TS 10974:2018 (E). (2018). Assessment of the safety of magnetic resonance imaging for patients with an active implantable medical device.

3. AAMI PC76 (Draft), "Requirements and Test Protocols for Safety of Patients with Pacemakers and ICDs Exposed to MRI", to be published.

4. Wilkoff, B. L., et al. (2013). Safe magnetic resonance imaging scanning of patients with cardiac rhythm devices: A role for computer modeling. Heart Rhythm, 10(12), 1815-1821.

5. Brown, J. E., et al. (2016). MR conditional safety assessment of implanted medical devices: Advantages of computational human phantoms. In: Proceedings of 38th Annual International Conference IEEE EMBC, Orlando, FL, pp. 6465-6468.

6. Park, S.-M., et al. (2007). Calculation of MRI-induced heating of an implanted medical Lead wire with an electric field transfer function. Journal of Magnetic Resonance Imaging, 26, $1278-1285$.

7. Feng, S., et al. (2015). A technique to evaluate MRI-induced electric fields at the ends of practical implanted lead. IEEE Transactions on Microwave Theory and Techniques, 63(1), 305-313.

8. Brown, J. E. (2012). Radiofrequency heating near medical devices in magnetic resonance imaging. Ph.D. dissertation, Bobby B. Lyle School of Engineering, Southern Methodist University, Dallas, TX.

9. Brown, J. E., \& Lee, C. S. (2013). Radiofrequency resonance heating near medical devices in magnetic resonance imaging. Microwave and Optical Technology Letters, 55, 2-299.

10. McCabe, S. O., \& Scott, J. B. (2014). Cause and amelioration of MRI-induced heating through medical implant lead wires. In 21 st electrical New Zealand conference, Hamilton, New Zealand.

11. McCabe, S. O., \& Scott, J. B. (2015). Technique to assess the compatibility of medical implants to the RF field in MRI. In Asia-Pacific Microwave Conference 2015, pp. 6-9.

12. Liu, J., et al. (2019). On the relationship between impedances of active implantable medical devices and device safety under MRI RF emission. In IEEE Transactions, EMC, Accepted (to be published).

13. Yao, A., et al. (2019). Efficient and reliable assessment of the maximum local tissue temperature increase at the electrodes of medical implants under MRI exposure. Bioelectromagnetics, 40(6), 422-433.

14. Kozlov, M., \& Kainz, W. (2017). Comparison of lead electromagnetic model and 3D EM results for helix and straight leads. In Proceedings of 19th International Confernce on Electromagnetic Advances and Applications, pp. 649-652.

15. Kozlov, M., \& Kainz, W. (2018). Lead electromagnetic model to evaluate RF-induced heating of a coax lead: A numerical case study at $128 \mathrm{MHz}$. IEEE Journal of Electromagnetics. RF and Microwaves in Medicine and Biology, 2(4), 286-293.

16. Zastrow, E., Capstick, M., \& Kuster, N. (2016).Experimental system for RF-heating characterization of medical implants during MRI. In Proceedings of 24th Annual Meeting ISMRM, Singapore.

17. Zastrow, E., Yao, A., \& Kuster, N. (2017). Practical considerations in experimental evaluations of RF-induced heating of leaded implants. In 32nd URSI GASS, Montreal, Canada. 
18. Brown, J. E., et al. (2018). Calculation of MRI RF-induced voltages for implanted medical devices using computational human models. In S. Makarov et al. (Eds.), Brain and human body modeling: Computational human modeling at EMBC 2018 (pp. 283-294). Cham: Springer Nature.

19. Brown, J. E., et al. (2018). Calculating RF-induced voltages for implanted medical devices in MRI using computational human models. In Proceedings of 40th annual international conference, IEEE EMBC, Honolulu, HI, pp. 3866-3869.

20. Coates, S., \& Thwaites, B. (2000). The strength-duration curve and its importance in pacing efficiency: A study of 235 pacing leads in 229 patients. PACE, 23, 1273-1277.

21. Kurpad, K., et al. (2018) MRI RF safety of active implantable medical devices (AIMDs): Effect of conductivity of tissue simulating media on device model accuracy. In Proceedings of 26th annual meeting international society of magnetic resonance in medicine, June 16-21, 2018, Paris, France, pp. 4075.

22. Min, X., \& Sison, S. (2017). Impact of mixed media on transfer functions with a pacemaker system for estimation of RF heating during MRI scans. Computers in Cardiology, 44, 1-4.

23. Min, X., \& Sison, S. (2018). Transfer functions of a spinal cord stimulation systems in mixed media and homogeneous media for estimation of RF heating during MRI Scans. In Proceedings of 40th annual international conference, IEEE EMBC, Honolulu, HI, pp. 2048-2051.

24. Kurpad, K. N., et al. (Accepted). MRI rf safety of active implantable medical devices (AIMDs): Tissue simulating medium properties for accurate modeling of MRI-conditional AIMDs. In 28th annual meeting international society of magnetic resonance in medicine.

25. Brown, J. E., et al. (Accepted). MRI safety of active implantable medical devices: Numerical study of the effect of lead insulation thickness on the RF-induced tissue heating at the lead electrode. In 42nd annual international conference, IEEE EMBC.

26. Noetscher, G. M., et al. (2016). Computational human model VHP-female derived from datasets of the National Library of Medicine. In Proceedings of 38th annual international conference, IEEE EMBC, Orlando, FL, pp. 3350-3353.

27. Ibrahim, T. S., et al. (2000). Application of finite difference time domain method for the design of birdcage RF head coils using multi-port excitations. Magnetic Resonance Imaging, 10, 733-742.

28. McElcheran, C. E., Golestanirad, L., Iacono, M. I., et al. (2019). Numerical simulations of realistic lead trajectories and an experimental verification support the efficacy of parallel radiofrequency transmission to reduce heating of deep brain stimulation implants during MRI. Scientific Reports, 9, 2124.

Open Access This chapter is licensed under the terms of the Creative Commons Attribution 4.0 International License (http://creativecommons.org/licenses/by/4.0/), which permits use, sharing, adaptation, distribution and reproduction in any medium or format, as long as you give appropriate credit to the original author(s) and the source, provide a link to the Creative Commons license and indicate if changes were made.

The images or other third party material in this chapter are included in the chapter's Creative Commons license, unless indicated otherwise in a credit line to the material. If material is not included in the chapter's Creative Commons license and your intended use is not permitted by statutory regulation or exceeds the permitted use, you will need to obtain permission directly from the copyright holder.

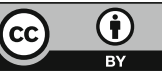

